\title{
Epoxy as a reactive plasticizer for improving polycarbonate processibility
}

\author{
Genhai G. Liang ${ }^{\mathrm{a}}$, Wayne D. Cook ${ }^{\mathrm{a}, *}$, Abbas Tcharkhtchi $^{\mathrm{b}}$, Henry Sautereau ${ }^{\mathrm{c}}$ \\ a Materials Engineering Dept., Monash University, Clayton, Vic 3800, Australia \\ ${ }^{\mathrm{b}}$ Laboratoire d'Ingénierie des Matériaux, ENSAM Paris, France \\ ${ }^{\mathrm{c}}$ Laboratoire des Matériaux Macromoléculaires, INSA de Lyon, France
}

Keywords:

Polycarbonate

Epoxy

Reactive plasticizer

Processibility

Crystallisation

Phase separation

\begin{abstract}
A B S T R A C T
An oligomer of a diepoxy (diglycidyl ether of bisphenol-A, DGEBA) and an aromatic diamine (MCDEA) have been used as reactive plasticizers for polycarbonate (PC). A small amount of PC chain scission occurred during this blending process, probably due to transesterification of the PC carbonate group by the hydroxyl group of the DGEBA oligomer. Addition of DGEBA to PC was found to greatly reduce the $T_{g}$ and processing temperature. Dynamic rheology measurements showed that the added epoxy can very effectively reduce the viscosity, but that the addition of epoxy also accelerated the crystallisation rate of the PC, which was confirmed by XRD, optical transmission microscopy and DMTA. The DMTA results of cured blends also showed that this crystallization of the PC enhanced their heat resistance properties. Sol-gel studies of the cured samples showed that some of the PC was grafted to the crosslinked epoxy network. Studies of the rubbery behaviour, solvent resistance of the cured blend and SEM images suggest that PC is the main continuous phase in the matrix and that the epoxy phase is mainly dispersed as sub-micron particles in the matrix.
\end{abstract}

\section{Introduction}

Bisphenol-A polycarbonate (PC) is one of the toughest and clearest plastics, but it is also difficult to process due to its high melt viscosity caused by the rigid chain structure, resulting in a relatively high processing temperatures (at least $280^{\circ} \mathrm{C}$ ) and/or high screw torques. As a result, side reactions such as the hydrolysis with traces of absorbed moisture may occur during the processing at the high temperature, causing property degradation. Traditional resolutions of these problems, such as reducing the PC molecular weight, adding inert plasticizers, and/or blending with a more processable polymer, tend to impair the mechanical and thermal properties of the final product. An alternative strategy has been developed for the processing of thermoplastics with high viscosity [1-14] in which the processing temperature is lowered by adding a small amount of crosslinkable monomers. These monomers act as reactive

\footnotetext{
* Corresponding author. Fax: +61 399054940.

E-mail address: wayne.cook@monash.edu (W.D. Cook).
}

plasticizers during processing by lowering the glass transition temperature and viscosity, but they can be subsequently polymerised into a thermoset or rubbery phase in the thermoplastic matrix, potentially retaining the original mechanical properties of the matrix. We have recently proposed a new application of this strategy to rotational moulding and powder coating [8] - due to the long cycle times involved in these processes, degradation of the thermoplastic is highly probable but if the processing temperature can be lowered by addition of a reactive plasticizer, this problem will be minimised.

Epoxy resins and diallylic monomers are the most common thermosetting monomers that have been used as reactive plasticizers for thermoplastics such as poly $(2,6-$ dimethyl-1,4-phenylene ether) (PPE) [1-3,5,15] and poly(methyl methacrylate) (PMMA) [8,16], because these monomers have a relatively low viscosities which aids the processing, high boiling temperatures to minimise evaporation, and low reactivities at high temperatures to prevent from the early-stage gelation during the processing. In addition, upon crosslinking these reactive plasticizers, the resulting crosslinked polymer may possess higher 
thermo-mechanical properties than the processed thermoplastic, which may improve the properties of the thermoplastic.

In this investigation we explore the possibility of enhancing the processing of PC using small amounts of an epoxy/diamine resin system, as a curable processing aid. Thus in the present study, the PC is the continuous phase, which contrasts with previous research $[17,18,22]$ where small amounts of PC were added as a dispersed phase in an epoxy resin to toughen the cured thermoset matrix. In addition we also investigate the crystallisation of PC induced by the unreacted epoxy resin and its effect on the thermal-resistance of the final cured PC/epoxy resin blend.

\section{Experimental}

\subsection{Materials}

PC pellets were obtained from GE Plastics (Australia) as Lexan HF1140-111. The epoxy selected for this work is based on the diglycidyl ether of bisphenol-A (DGEBA) and this was supplied in two grades. Ciba-Geigy kindly supplied the first grade of DGEBA (Araldite GY9708-1) which was used in the initial studies of PC/DGEBA blends and this material had a reported epoxy equivalent weight of $190 \mathrm{~g} /$ mol and so the fraction ( $n$, see in Fig. 1) of the bisphenol-A glycerol unit in the oligomer is about 0.15 - this oligomer is described here as $\operatorname{DGEBA}(n=0.15)$. The second grade was kindly supplied by DOW Chemical as DER 332 and noted here as $\operatorname{DGEBA}(n=0.04)$ and this had an epoxy equivalent weight of 176 and so the fraction, $n$, is about 0.04 . This second grade of DGEBA was selected when it was recognised that interchange reactions could occur between the oligomer's hydroxyl group and the PC carbonate unit. $\operatorname{DGEBA}(n=0.04)$ was cured with either a stoichiometric amount of 4,4'-methylene-bis(3-chloro-2,6-diethylaniline) (MCDEA, Sigma-Aldrich) or hexahydrophthalic anhydride (HHPA, supplied by Aldrich) containing $1 \mathrm{wt} . \%$ dimethyl benzylamine (DMBA, supplied by Aldrich). All raw materials were used without further treatments, and their chemical structures are listed in Table 1. The samples

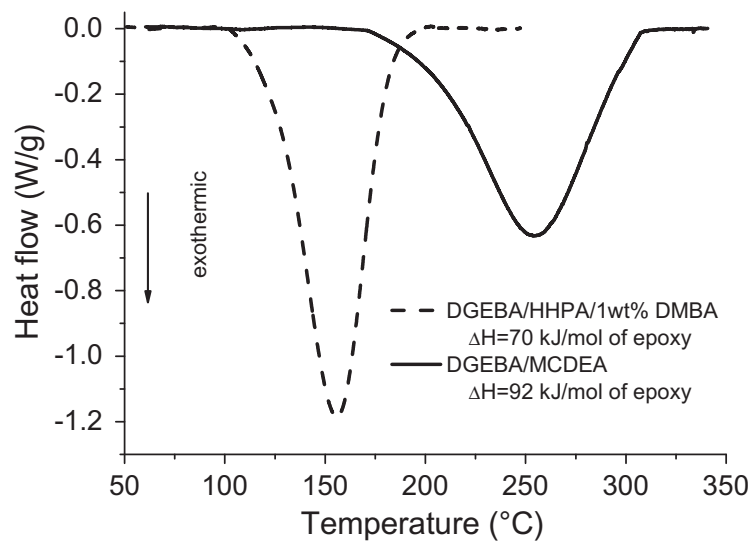

Fig. 1. Temperature-ramped DSC curing of $\operatorname{DGEBA}(n=0.04)$ with MCDEA or HHPA at a heating rate of $10^{\circ} \mathrm{C} / \mathrm{min}$. are identified by a code giving the weight ratios of $\mathrm{PC}$ and epoxy-hardener - thus 70/30 PC/DGEBA( $n=0.04)$-MCDEA designates a sample with $70 \mathrm{wt} . \%$ PC and $30 \mathrm{wt} . \%$ of a stoichiometric ratio of DGEBA $(n=0.04)$ and MCDEA.

\subsection{Sample preparation}

For studies of PC/DGEBA blends, PC pellets were manually pre-mixed with the $\operatorname{DGEBA}(n=0.15)$ resin at ratios of $100 / 0,90 / 10,80 / 20,70 / 30$ and $60 / 40$, and were set aside overnight so that the PC could absorb the epoxy resin. This PC/DGEBA mixture was then added into the hopper of a micro-extruder (Thermo Electron, Haake MiniLab, Germany) and was extruded by a pair of tapered screws $(110 \mathrm{~mm}$ long with the diameter linearly tapering from $10 \mathrm{~mm}$ to $5 \mathrm{~mm}$ ). The screw speed was $100 \mathrm{rpm}$, the duration of the processing operation was about $1 \mathrm{~min}$, and the pre-set temperature of the barrel, recycling channel and die ranged from $280^{\circ} \mathrm{C}$ for neat $\mathrm{PC}, 250^{\circ} \mathrm{C}$ for the PC/DGEBA $(90 / 20, w / w)$ blend, $230{ }^{\circ} \mathrm{C}$ for $80 / 20$ PC/DGEBA, $210^{\circ} \mathrm{C}$ for $70 / 30$ PC/DGEBA and $200^{\circ} \mathrm{C}$ for 60/40 PC/DGEBA. Under these conditions, the torque used to extrude the blends was approximately constant. The blend extrudate was immediately transferred into a micro-injection moulding machine (DSM Research, Netherland) pre-set the same temperature as the extruder and was then injected to a dog-bone steel mould at room temperature with dimensions of $50 \mathrm{~mm} \times 5 \mathrm{~mm} \times 2 \mathrm{~mm}$ in the middle section of the dog-bone sample. For the PC/DGEBA-MCDEA blends, the DGEBA $(n=0.04)$ resin was mixed with MCDEA at $c a$. $110{ }^{\circ} \mathrm{C}$ then rapidly cooled to room temperature. This DGEBA-MCDEA solution was then mixed with PC pellets manually and this blend stored overnight at room temperature to allow swelling of the pellets. Each PC/DGEBA-MCDEA blend was then extruded under the same conditions as employed for the same wt.\% PC in the PC/DGEBA blends. The extrudate had a diameter of about $2 \mathrm{~mm}$ and this was used for testing. All blended samples had a transparent appearance, suggesting miscibility between the blend components based on the observation that the refractive index of 1.590 for PC [19] is quite different from the value of 1.568 for DGEBA [20].

\subsection{Instrumental}

The molecular weight distributions of the PC and a 60/ $40 \mathrm{PC} / \operatorname{DGEBA}(n=0.15)$ blend were measured by a Waters (USA) gel permeation chromatography (GPC) system with $100 \AA$ and $1000 \AA$ Styragel columns using tetrahydrofuran (THF) as solvent at a flow rate of $1 \mathrm{ml} / \mathrm{min}$ and a Sedex 55 (Sedere, France) evaporative light scattering detector. The GPC was calibrated using polystyrene standards and so the molecular weights reported here are in polystyrene equivalents.

Differential scanning calorimetry (DSC) curing studies of $\operatorname{DGEBA}(n=0.04)$ with MCDEA and HHPA were performed using a Perkin Elmer Pyris 1 with a Flexicool (ETS system) cooling system on $\sim 10 \mathrm{mg}$ samples sealed in aluminium pans and heated at $10^{\circ} \mathrm{C}$ per min from $50{ }^{\circ} \mathrm{C}$ to $200{ }^{\circ} \mathrm{C}$ under a $20 \mathrm{ml} / \mathrm{min}$ nitrogen purge. DSC was also used to measure the $T_{g}$ of $\operatorname{DGEBA}(n=0.15)$ as $-14^{\circ} \mathrm{C}$, with 
Table 1

Raw materials used.

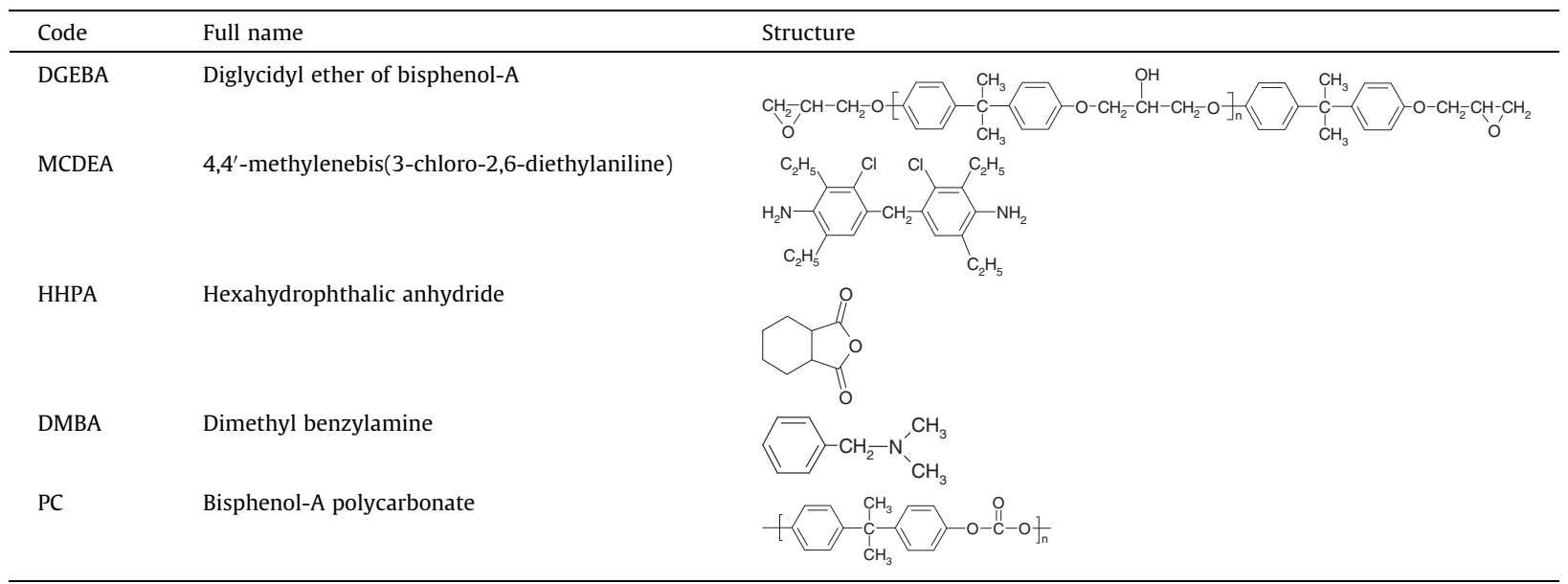

a heat capacity step of $0.61 \mathrm{~J} / \mathrm{g} / \mathrm{K}$. DSC experiments were also used to measure the crystallinity of $\sim 10 \mathrm{mg}$ samples of the specimens which had been tested by dynamic mechanical thermal analysis (see below).

The temperature dependence of the rheological properties of $\operatorname{PC} / \operatorname{DGEBA}(n=0.15)$ blends were measured in dynamic shear modes using an ARES rheometer (Rheometrics Scientific, USA) between parallel circular plates (diameters ranging from 15 to $40 \mathrm{~mm}$ depending on the viscosity of the blend being investigated) with a $0.5 \mathrm{~mm}$ gap. The real $\left(G^{\prime}\right)$ and imaginary $\left(G^{\prime \prime}\right)$ shear moduli were measured as a function of temperature by cooling the molten sample down from either $250^{\circ} \mathrm{C}$ or $200^{\circ} \mathrm{C}$ (depending on the sample viscosity) to approximately $145^{\circ} \mathrm{C}$ (depending on the rigidity of the blend) at $10^{\circ} \mathrm{C} /$ min while varying the frequency from 0.1 to $10 \mathrm{~Hz}$ at an average strain of $5 \%$.

Optical transmission microscopy studies of phase separation during the cure of $\operatorname{PC} / \operatorname{DGEBA}(n=0.04)-\mathrm{MCDEA}$ blends was undertaken by sandwiching the extruded uncured blend between a microscope slide and a cover slip so that the sample thickness was $\sim 0.1 \mathrm{~mm}$, followed by an immediate quench in ice-water, thus resulting in a transparent amorphous sample. The sample was mounted on a Mettler FP80 hotstage (Switzerland) attached to an Olympus (Japan) BH-1 optical microscope and was heated from $25^{\circ} \mathrm{C}$ to $270{ }^{\circ} \mathrm{C}$ at the rate of $5^{\circ} \mathrm{C} / \mathrm{min}$ while it was illuminated with non-polarised light. The transmitted light passing through the sandwiched sample was collected by a Mettler ZU FP80 light sensor (Switzerland) and electronically recorded as a function of the temperature. The light intensity at the start of the experiment was used to normalise the data.

A dynamic mechanical thermal analyser (DMTA Mark IV, Rheometric Scientific, USA) was used in dual cantilever bending mode to measure the real and loss flexural moduli over the temperature range from $-100^{\circ} \mathrm{C}$ to $150^{\circ} \mathrm{C}$ at $2{ }^{\circ} \mathrm{C}$ per min at $1 \mathrm{~Hz}$ with bar-type specimen of dimension $20 \mathrm{~mm} \times 5 \mathrm{~mm} \times 1.5 \mathrm{~mm} \quad(L \times W \times H) \quad$ for $\quad$ PC/DGE$\mathrm{BA}(n=0.15)$ samples and rod-shaped specimens (diameter $\sim 2 \mathrm{~mm})$ for $\operatorname{PC} / \operatorname{DGEBA}(n=0.04)-\mathrm{MCDEA}$ samples. The materials studied were uncured $\operatorname{PC} / \operatorname{DGEBA}(n=0.15)$ blends and PC/DGEBA $(n=0.04)-$ MCDEA mixtures which had been cured at $150{ }^{\circ} \mathrm{C}$ for $20 \mathrm{~h}$ (and in one case at $200{ }^{\circ} \mathrm{C} / 20 \mathrm{~h}$ ). At the end of the DMTA experiment the samples were cooled at approximately $50{ }^{\circ} \mathrm{C} / \mathrm{min}$ to room temperature. In some cases the DMTA experiment was repeated on the same specimen a second time to determine whether any changes had occurred in the material. The glass transition temperature $\left(T_{g}\right)$ was defined as the temperature corresponding to the maximum in $\tan \delta$.

The wide angle X-ray diffraction (XRD) patterns were measured at room temperature on uncured and cured $\operatorname{PC} / \operatorname{DGEBA}(n=0.04)-\mathrm{MCDEA}$ blends in a Philips XRD instrument under reflection mode with the measurement scan rate at $2^{\circ}$ per min from $2^{\circ}$ to $60^{\circ}$ using step size of $0.02^{\circ}$. An acceleration voltage of $40 \mathrm{kV}$ and current of $25 \mathrm{~mA}$ were applied to generate a Ni-filtered $\mathrm{Cu}_{-} \mathrm{K}_{\alpha}$ radiation of $0.154 \mathrm{~nm}$ in wavelength.

To determine whether any of the PC was grafted into the epoxy network in the blends, extraction studies were performed on finely ground samples of PC/DGEBA $(n=0.04)-$ MCDEA (cured at $150{ }^{\circ} \mathrm{C} / 20 \mathrm{~h}$ and postcured at $200^{\circ} \mathrm{C} /$ $30 \mathrm{~min}$ ) with dichloromethane for $15 \mathrm{~h}$ using an extraction thimble and a Soxhlet extractor. To determine whether the epoxy network phase was co-continuous in the PC/DGEBA-MCDEA blends, samples were immersed in dichloromethane for three months and their dissolution, disintegration or swelling was monitored by visual inspection.

The SEM images were taken with JEOL JSM-6300F scanning electron microscope at $15 \mathrm{kV}$ on the gold-coated surface of the freeze-fractured blends.

\section{Results and discussions}

\subsection{The DGEBA hardener selection and the reaction between $P C$ and DGEBA}

MCDEA and HHPA were investigated as potential curing agents of DEGBA-plasticized PC. Fig. 1 shows that DGE$\mathrm{BA}(n=0.04)-\mathrm{MCDEA}$ exhibits a higher temperature DSC 
exotherm than DGEBA-HHPA, indicating it cures more slowly. This suggests that MCDEA is a more suitable epoxy hardener than the anhydride harder, because it can avoid the pre-curing of epoxy during processing of the $\mathrm{PC} /$ epoxy-hardener blend at the elevated temperature. In addition, the heat of polymerisation for DGEBA $(n=0.04)-$ MCDEA (92 kJ/mol of epoxy) compares well with values ranging from $100-118 \mathrm{~kJ} / \mathrm{mol}$ for aromatic epoxy/amine reactions tabulated in a review by Rozenberg [21], and is greater than that measured for $\operatorname{DGEBA}(n=0.04) / \mathrm{HHPA} /$ $1 \mathrm{wt} . \%$ DMBA ( $70 \mathrm{~kJ} / \mathrm{mol}$ of epoxy), which is also an advantage because it means a higher extent of reaction is attained with the former. The data in Fig. 1 can be used to make an approximate estimate of how much polymerisation could occur during processing of $P C$ with the polymerizable $\operatorname{DGEBA}(n=0.04)$ and MCDEA monomers. Assuming that the dilution of $\operatorname{DGEBA}(n=0.04)-$ MCDEA by PC does not slow the reaction (it would be expected to do so because the concentration of reacting groups is reduced) then multiplication of the heat flow at each of the processing temperatures (see Section 2) by the processing time (60 s) and division by the total heat of reaction shows that the extent of reaction in the 90/10 $\operatorname{PC} / \operatorname{DGEBA}(n=0.04)-$ MCDEA blend would be about $14 \%$ and less than $10 \%$ for the other blends and so can be ignored.

The chemical reaction between PC and DGEBA $(n=0.15)$ was investigated using GPC. The virgin PC had a number average molecular weight $\left(\bar{M}_{n}\right)$ and weight average molecular weight $\left(\bar{M}_{w}\right)$ of 32,300 and $46,000 \mathrm{~g} / \mathrm{mol}$, respectively, but after extrusion and injection moulding at $280{ }^{\circ} \mathrm{C}$ for $5 \mathrm{~min}$ these values dropped to 30,000 and $42,300 \mathrm{~g} / \mathrm{mol}$, respectively, possibly resulting from the hydrolysis with traces of adventitious water. After the processing of the $60 / 40 \operatorname{PC} / \operatorname{DGEBA}(n=0.15)$ blend at $210^{\circ} \mathrm{C}$ for $5 \mathrm{~min}$, the molecular weights were further reduced to a $\bar{M}_{n}$ and $\bar{M}_{w}$ of 26,200 and $35,900 \mathrm{~g} / \mathrm{mol}$, respectively. Similar results were obtained in studies of Don and Bell [22] on a similar DGEBA oligomer and PC. These data suggest that bond interchange reactions, i.e. transesterification, had occurred between the epoxy's hydroxyl group and the PC carbonate group, as schematically shown in Scheme 1. Since the DGE$\mathrm{BA}(n=0.15)$ oligomer has only $15 \mathrm{~mol} \%$ of the glycerol unit (see Table 1), statistically, the dominant species will be DGEBA monomer ( $85 \mathrm{~mol} \%$ ) and the $n=1$ oligomer (15\%). Based on the observed reduction in PC molecular weight, the stoichiometry of the reaction (see Scheme 1), mass and mole balances, and assuming that the vast majority of PC molecules are only cleaved once, the fraction of the $n=1$ oligomer which reacts with $\mathrm{PC}$ is calculated to be 0.027 and the fraction of PC chains which are cleaved is 0.24 (this justifies the assumption of single scission). However it should be noted that simple stoichiometry calculations show that if all of the hydroxyl groups in $\operatorname{DGEBA}(n=0.15)$ underwent bond interchange with the PC carbonate units, every PC chain would be scissioned. Therefore to reduce the extent of bond interchange, for the studies of PC with DGEBA oligomer and MCDEA, we have concentrated on the oligomer $\operatorname{DGEBA}(n=0.04)$ which would be much less likely to undergo bond interchange with PC because only $4 \%$ of the epoxy molecules contain hydroxyl groups. However, since the curing of DGEBA with an amine (MCDEA) produces hydroxyl groups, further levels of bong interchange are possible during extended cure cycles.

\subsection{Processibility enhancement due to DGEBA plasticization of $P C$}

As mentioned above and illustrated in Fig. 2, the addition of $\operatorname{DGEBA}(n=0.15)$ to PC significantly decreases the<smiles>CC(C)(c1ccc(OCC(O)COc2ccc(C(C)(C)c3ccc(C(C)(C)c4ccc(C(C)(C)c5ccc(OCC6CO6)cc5)cc4)cc3)cc2)cc1)c1ccc(OCC2CO2)cc1</smiles>

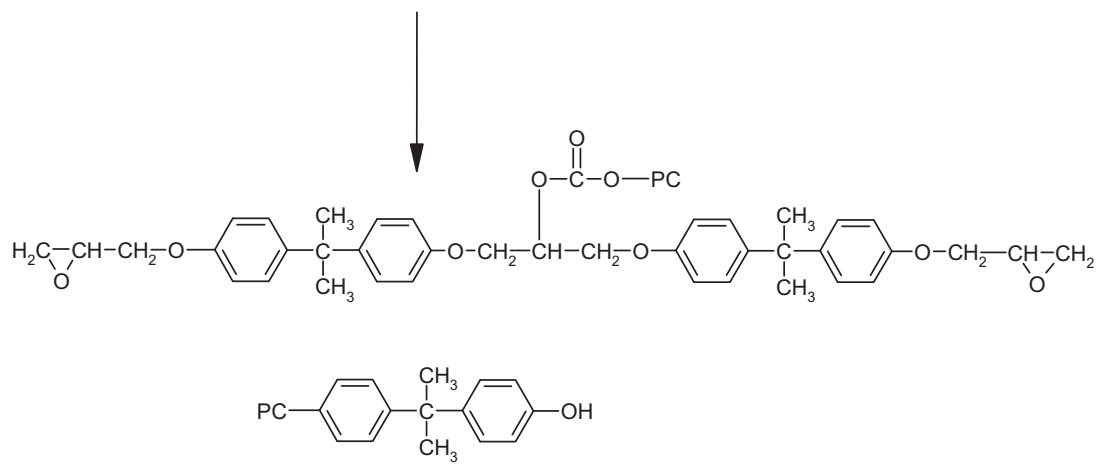

Scheme 1. The chain scission reaction between PC and the DGEBA oligomer (here showing the reaction for the oligomer with $n=1$ ). Note that for each reacted hydroxyl from this oligomer, two molecules are formed containing polycarbonate fragments and that the total number of polycarbonate-containing molecules increases by one. 


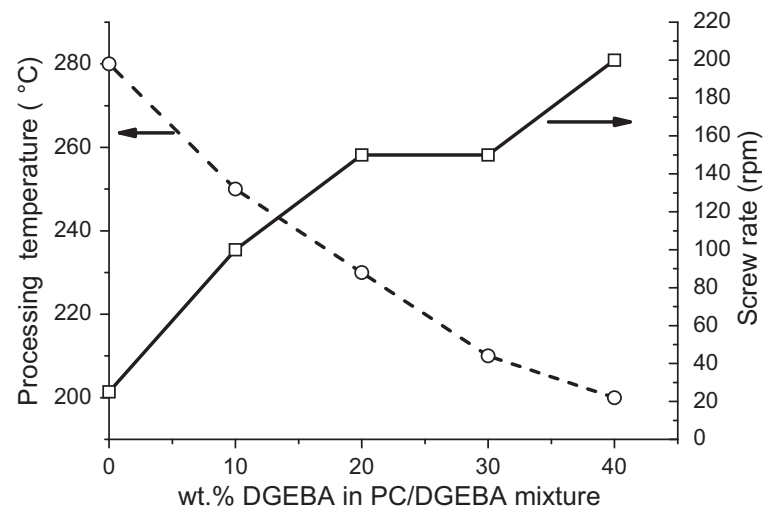

Fig. 2. Processing conditions of various $\operatorname{PC} / \mathrm{DGEBA}(n=0.15)$ melt blends.

temperature and increases the productivity without extra power (torque) required for the processing. This improvement of processibility was also observed in the preparation of $\operatorname{PC} / \operatorname{DGEBA}(n=0.04)$-MCDEA samples. This can be interpreted in terms of the ability of DGEBA to increase the lubrication of the PC chains as they slide past one another, thus reducing the blend's viscosity. A lower viscosity can allow the processing rate to be raised so that the productivity is improved, In addition, a reduction of the processing temperature means that degradative side reactions can be effectively reduced or even eliminated.

The dynamic rheology of PC/DGEBA $(n=0.15)$ blends at $1 \mathrm{~Hz}$ (similar data was obtained at 0.1 and $10 \mathrm{~Hz}$ ), as the melt was cooled, is shown in Fig. 3. These data reveal that the addition of the DGEBA epoxy resin significantly reduces the storage and loss moduli of the PC/DGEBA blends, and since the dynamic viscosity $\left(\eta^{\prime}\right)$ is proportional to the loss modulus $\left(\eta^{\prime}=G^{\prime \prime} / \omega\right.$ where $\omega$ is the oscillatory frequency) the viscosity is also reduced by up to three decades (depending on the temperature), as indicated by the

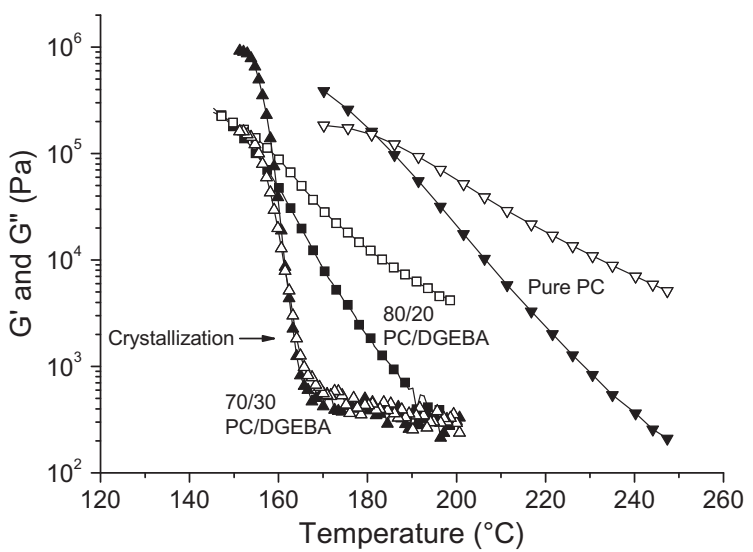

Fig. 3. Real (solid symbols) and loss (open symbols) shear moduli at $1 \mathrm{~Hz}$ of $70 / 30(\Lambda, \triangle)$ and $80 / 20(\boldsymbol{\square}, \square)$ PC/DGEBA $(n=0.15)$ blends and pure PC $(\boldsymbol{\nabla}, \nabla)$ as a function of temperature. data in Fig. 2. This suggests that at the same processing pressures and stresses, the processing temperature could be further reduced by the addition of more DGEBA to the PC. However during studies of the effect of differing extrusion temperatures on the processing of the 70/30 $\operatorname{PC} / \operatorname{DGEBA}(n=0.15)$ blend, it was found below a critical temperature the extruder torque was suddenly overloaded, caused by the solidification of the blend melt near the extruder die exit. The blend which was blocking the extruder nozzle appeared opaque, rather than the transparency observed when processing above this critical temperature. This change in viscosity and transparency can be ascribed to crystallisation of the PC from the PC/DGEBA melt. Under non-plasticized processing of PC, crystallization is not commonly observed industrially due to the high melt viscosity of PC and the short processing time involved which does not permit the chains to crystallize before the polymer is cooled below its glass transition. However in solution (such as in DGEBA here) the PC's rigid chain is provided greater mobility, thus allowing faster re-arrangement into the thermodynamically preferred crystalline form [19], and this has been reported by Don and Bell [22] for low concentrations of PC in an epoxy resin. Fig. 3 also clearly shows that the $G^{\prime}$ and $G^{\prime \prime}$ values of the 70/30 $\operatorname{PC} / \operatorname{DGEBA}(n=0.15)$ blend suddenly increased when the temperature decreased to this critical temperature, whereas the pure PC and the 80/20 PC/DGEBA $(n=0.15)$ blend only showed a steady increase in moduli - this suggests that due to the reduced molecular mobility and limited timescale of the experiment no crystallisation had occurred for these last systems. This conclusion is also consistent with the observations that the samples of pure PC and $80 / 20 \operatorname{PC} / \operatorname{DGEBA}(n=0.15)$ were still transparent after the dynamic rheology experiments, while the 70/30 PC/ $\operatorname{DGEBA}(n=0.15)$ sample had became opaque. It was also noted that the samples with relatively higher amounts of $\operatorname{DGEBA}(n=0.15)$ or DGEBA-MCDEA $(n=0.04)$ easily crystallised during the extrusion process if the manufacturing temperature was reduced below a certain temperature. Thus the occurrence of recrystallization of $\mathrm{PC}$ in the blends during the processing of the PC/DGEBA blends limits the extent to which the processing temperature can be lowered.

DMTA studies of the PC/DGEBA $(n=0.15)$ blends in the as-extruded and injection moulded form (Fig. 4) show that each had only one major glass transition temperature $\left(T_{g}\right)$, indicating that the PC and DGEBA were miscible which is consistent with their transparent appearance. The $T_{g}$ of the blend decreases with the addition of DGEBA (see Fig. 5), and this trend is compared with that predicted by the Fox equation [23]:

$\frac{1}{T_{g}}=\frac{W_{P C}}{T_{g, P C}}+\frac{W_{D G E B A}}{T_{g, D G E B A}}$

where $W_{P C}$ and $W_{D G E B A}$ are weight fractions of PC and DGE$\mathrm{BA}$, respectively, and $T_{g, P C}$ and $T_{g, D G E B A}$ are the $T_{g}$ values (in $\mathrm{K})$ of $\mathrm{PC}\left(150^{\circ} \mathrm{C}\right)$ and $\operatorname{DGEBA}(n=0.15)\left(-14^{\circ} \mathrm{C}\right)$, respectively. This calculation (see the full line in Fig. 5) assumes that no chain scission of PC occurred by reaction of the $\operatorname{DGEBA}(n=0.15)$ oligomer hydroxy groups whereas the 


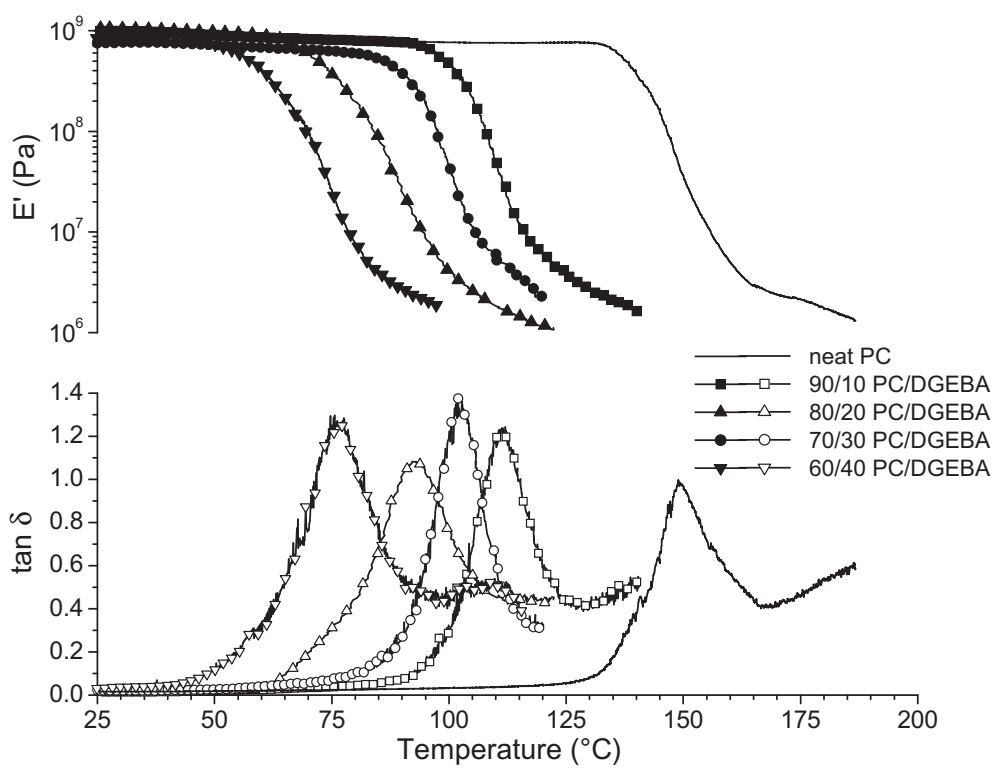

Fig. 4. DMTA of various uncured $\operatorname{PC} / \operatorname{DGEBA}(n=0.15)$ blends. Data beyond the "rubbery plateau" are omitted for clarity and are presented below.

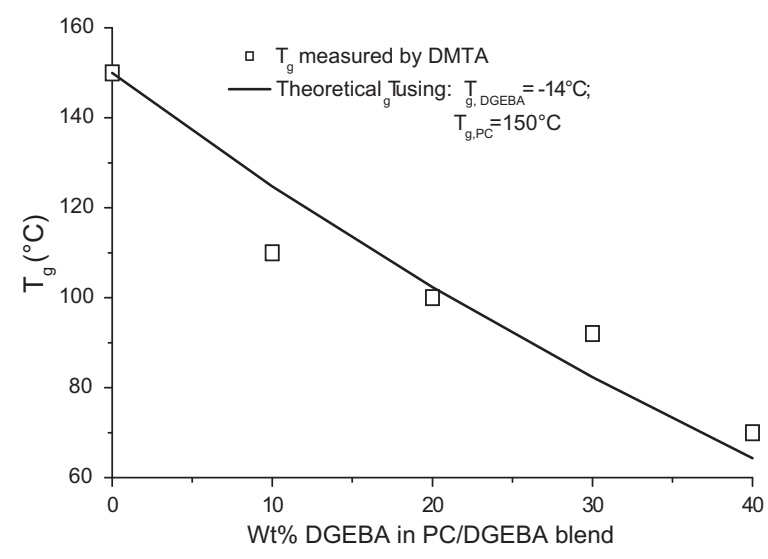

Fig. 5. $T_{g}$ of various $\operatorname{PC} / \operatorname{DGEBA}(n=0.15)$ blends compared with the theoretical $T_{g}$ predicted for the blends in which the PC is assumed to have limited chain scission by the epoxy resin as calculated from GPC data.

GPC data previously presented above shows that the $\bar{M}_{n}$ was lowered from $30,000 \mathrm{~g} / \mathrm{mol}$ for the processed PC to $26,200 \mathrm{~g} / \mathrm{mol}$ for the processed $60 / 40 \mathrm{PC} / \mathrm{DGEBA}(n=0.15)$ blend, presumably due to chain scission (see Scheme 1). Use of the Fox-Flory equation [24,25]

$T_{g}=T_{g, \infty}-\frac{K}{\bar{M}_{n}}$

where $T_{g, \infty}$ is the glass transition temperature for an ideal chain of infinite length, and $K\left(2.4 \times 10^{5} \mathrm{~g} / \mathrm{mol}\right.$ [26]) is a constant, shows that this reduction in PC molecular weight would only cause a $1.5^{\circ} \mathrm{C}$ reduction in $T_{g}$. The difference between the predicted line and the data is far greater than this, but the reason for this is not understood.

\subsection{PC crystallisation induced by the epoxy oligomer}

As shown in Fig. 2 and observed during the processing of the blends, the DGEBA acts not only as a plasticiser to enhance the processability of PC, but also as a solvent to induce crystallisation of the PC. During the DMTA measurements of the PC/DGEBA $(n=0.15)$ blends, it was observed that after the glass transition region, the modulus rose substantially for the uncured but quenched $70 / 30$ and $60 / 40$ $\operatorname{PC} / \operatorname{DGEBA}(n=0.15)$ blends, as illustrated in Fig. 6 . Before the DMTA experiment, the quenched blends were transparent, suggesting an amorphous structure, but after temperature ramping in the DMTA, followed by slow cooling, the samples exhibited an opaque appearance. This phenomenon can be attributed to recrystallization of the PC from the DGEBA solvent when the temperature and time in the DMTA instrument provided sufficient mobility for the PC chains to align themselves. For the 70/30 PC/DGE$\mathrm{BA}(n=0.15)$ specimen which had been temperatureramped during the DMTA experiment, a subsequent DSC temperature ramping experiment (see Fig. 7) revealed the presence of crystallinity with a heat of fusion of $36 \mathrm{~J} /$ $\mathrm{g}$ (expressed as per unit mass of PC). This values compares with values reported by Sohn et al. [27] of up to $34 \mathrm{~J} / \mathrm{g}$ for slow crystallization of PC from the glassy state and the value of $110 \mathrm{~J} / \mathrm{g}$ by Mercier and Legras [28] for pure crystalline PC. The heats of fusion measured for the other specimens after the DMTA experiment are listed on Table 2 and this shows that the PC crystallinity was higher for greater DGEBA content because the uncured epoxy acts as a solvent and accelerates the crystallization of the PC.

This crystallisation induced by DGEBA (and also possibly MCDEA) was also demonstrated by using hot-stage microscopy of PC/DGEBA $(n=0.04) /$ MCDEA blends (see Fig. 8 ). It can be seen that the quenched blend of $P C$ and uncured $\operatorname{DGEBA}(n=0.04)$ and MCDEA at room temperature is amor- 


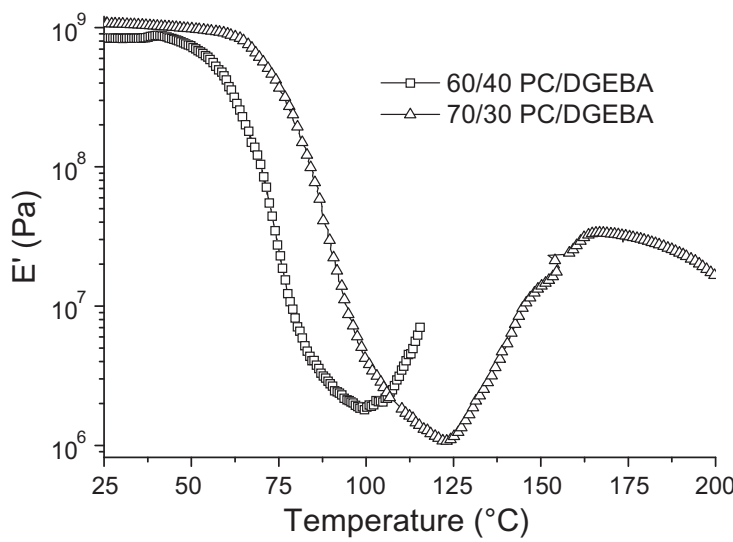

Fig. 6. Storage modulus versus temperature of quenched and amorphous blends of $60 / 40$ and 70/30 PC/DGEBA $(n=0.15)$.

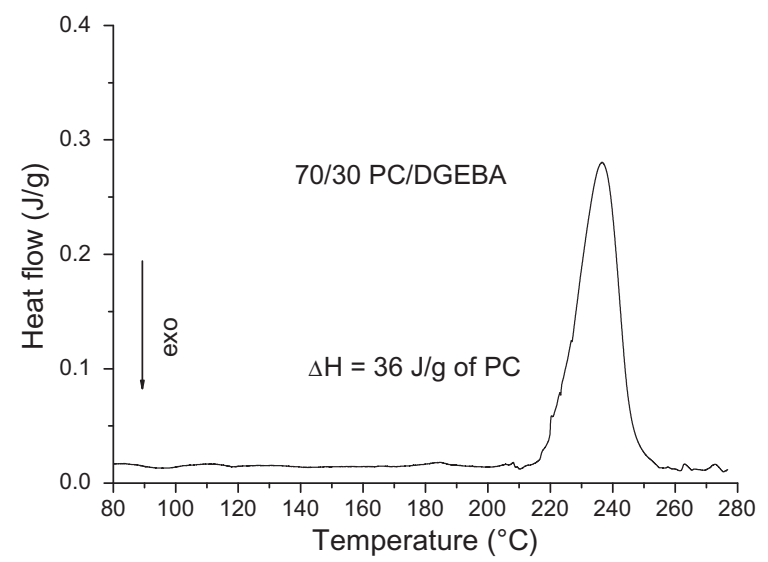

Fig. 7. DSC at $10^{\circ} \mathrm{C} / \mathrm{min}$ of crystallized $70 / 30 \operatorname{PC} / \operatorname{DGEBA}(n=0.15)$ blend after cooling the specimen which had been investigated by DMTA (see Fig. 6).

Table 2

Heat of fusion of various uncured $\operatorname{PC} / \operatorname{DGEBA}(n=0.15)$ blend specimens after they had been temperature ramped in the DMTA to temperatures and then cooled (see Fig. 4)

\begin{tabular}{lrrr} 
PC/DGEBA $(\mathrm{w} / \mathrm{w})$ & $80 / 20$ & $70 / 30$ & $60 / 40$ \\
Heat of fusion $(\mathrm{J} / \mathrm{g}$ of PC) & 28 & 36 & 46 \\
\hline
\end{tabular}

phous so the light transmission is at the maximum. When the temperature was raised to the PC's crystallisation temperature around $120^{\circ} \mathrm{C}$, some of the PC crystallized, resulting in scattering at the boundaries of the amorphous and crystalline regions so that the transparency decreased. On further temperature elevation up to $200^{\circ} \mathrm{C}$, the crystalline regions melted and so the transparency approached the maximum plateau again. However, based on the similarity in heating rates in the hot-stage microscopy $\left(5^{\circ} \mathrm{C} / \mathrm{min}\right.$, see Fig. 8) and DSC $\left(10^{\circ} \mathrm{C} / \mathrm{min}\right.$, see Fig. 1) experiments, the DSC data implies that during this temperature ramp in the hot-stage microscope, the DGEBA $(n=0.04)$-MCDEA would commence polymerisation at $\sim 170^{\circ} \mathrm{C}$ and reach a maximum rate of cure near $260^{\circ} \mathrm{C}$, so that after temperature ramping in the hot-stage microscope, the final blend should be a mixture of predominantly amorphous PC and cured

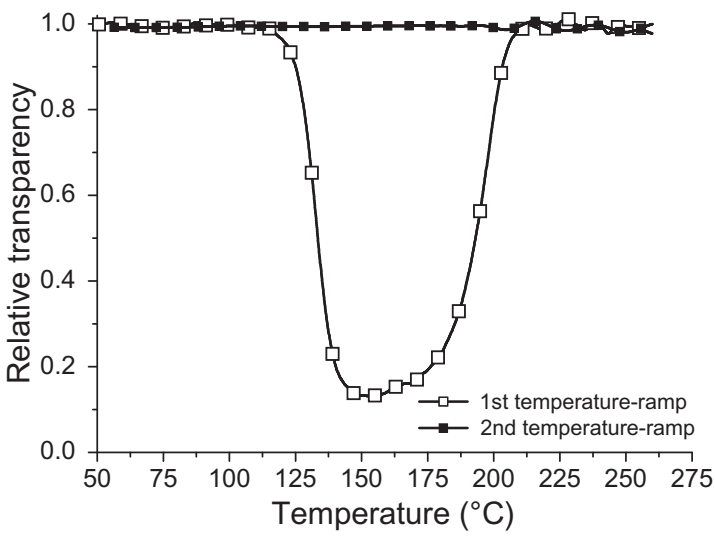

Fig. 8. Relative transparency of quenched 70/30 PC/DGEBA $(n=0.04)-$ MCDEA blend during the first temperature ramping in the hot-stage microscope at $5{ }^{\circ} \mathrm{C}$ per min to $260^{\circ} \mathrm{C}$, and then the same sample after rapid cooling and re-ramping at $5{ }^{\circ} \mathrm{C}$ per min. (some data points are omitted for clarity).

epoxy. When this sample was slowly cooled and then exposed to a second temperature-ramp in the microscope hot-stage, it was found that the sample's transparency remained high because the curing of the epoxy system could not longer provide molecular mobility to the PC and thus assist the crystallization.

The PC crystallisation induced by the uncured epoxy was also detected by using XRD. As shown in Fig. 9, only an amorphous broad peak is clearly observed for the extruded but quenched and uncured $\operatorname{PC} / \operatorname{DGEBA}(n=0.04)$ MCDEA blends (see (A), (B) and (C) in Fig. 9) because the quenching did not provide sufficient time for PC crystallization as discussed above. However, when the specimen was held at $150^{\circ} \mathrm{C}$ for $20 \mathrm{~h}$ to cure the epoxy component in the blend and the specimen then cooled, the XRD shows sharp peaks (see (a), (b) and (c) in Fig. 9) at $2 \theta=17.3^{\circ}$ (strong) and $23^{\circ}$ (weak), corresponding to a lattice spacing of $0.51 \mathrm{~nm}$ and $0.39 \mathrm{~nm}$, respectively in crystalline PC, as observed by Liao et al. [29]. This indicates that the PC component had undergone crystallisation during the early stages at $150{ }^{\circ} \mathrm{C}$, before the epoxy had fully cured. However, when the cured 70/30 PC/DGEBA $(n=0.04)$-MCDEA sample was temperature ramped in the DMTA from room temperature up to $250^{\circ} \mathrm{C}$ (see Fig. 10), the crystalline regions melted but did not reform when the specimen was again cooled to room temperature as shown by the XRD pattern in Fig. 9 (see curve (d)). It appears that insignificant crystallization occurred in the cured sample due to the absence of a solvent effect of the $\operatorname{DGEBA}(n=0.04)$ and MCDEA monomers on the mobility of the PC chains. This behaviour is analogous to what occurs when pure PC is rapidly cooled from the melt - the limited chain mobility and the lack of a very slow cooling process does not permit sufficient chain rearrangement for significant crystallization.

\subsection{Thermal-mechanical properties of cured $P C /$ $\operatorname{DGEBA}(n=0.04)-M C D E A$}

As discussed above, the PC/DGEBA $(n=0.04)-\operatorname{MCDEA}$ blends which had been cured at $150{ }^{\circ} \mathrm{C} / 20 \mathrm{~h}$ consisted of 


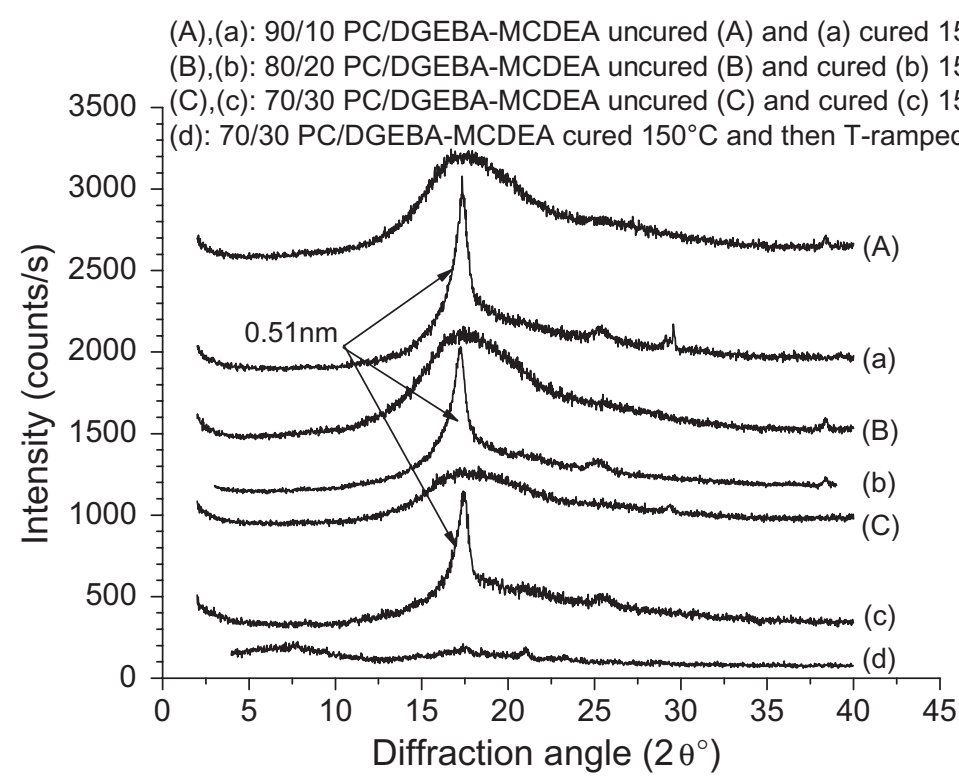

Fig. 9. Room temperature XRD of uncured PC/DGEBA $(n=0.04)-\mathrm{MCDEA}$ blends $(\mathrm{A}, \mathrm{B}$ and $\mathrm{C})$ and after curing at $150{ }^{\circ} \mathrm{C}$ for $20 \mathrm{~h}(\mathrm{a}$, b and $\mathrm{c})$. Also shown $(\mathrm{d})$ is the XRD behaviour of the $70 / 30 \mathrm{PC} / \mathrm{DGEBA}(n=0.04)-\mathrm{MCDEA}$ blend after curing at $150{ }^{\circ} \mathrm{C} / 20 \mathrm{~h}$, cooling to room temperature and then heating up to $250{ }^{\circ} \mathrm{C}$ in the DMTA, followed by rapidly cooling to room temperature and then conducting the XRD test.

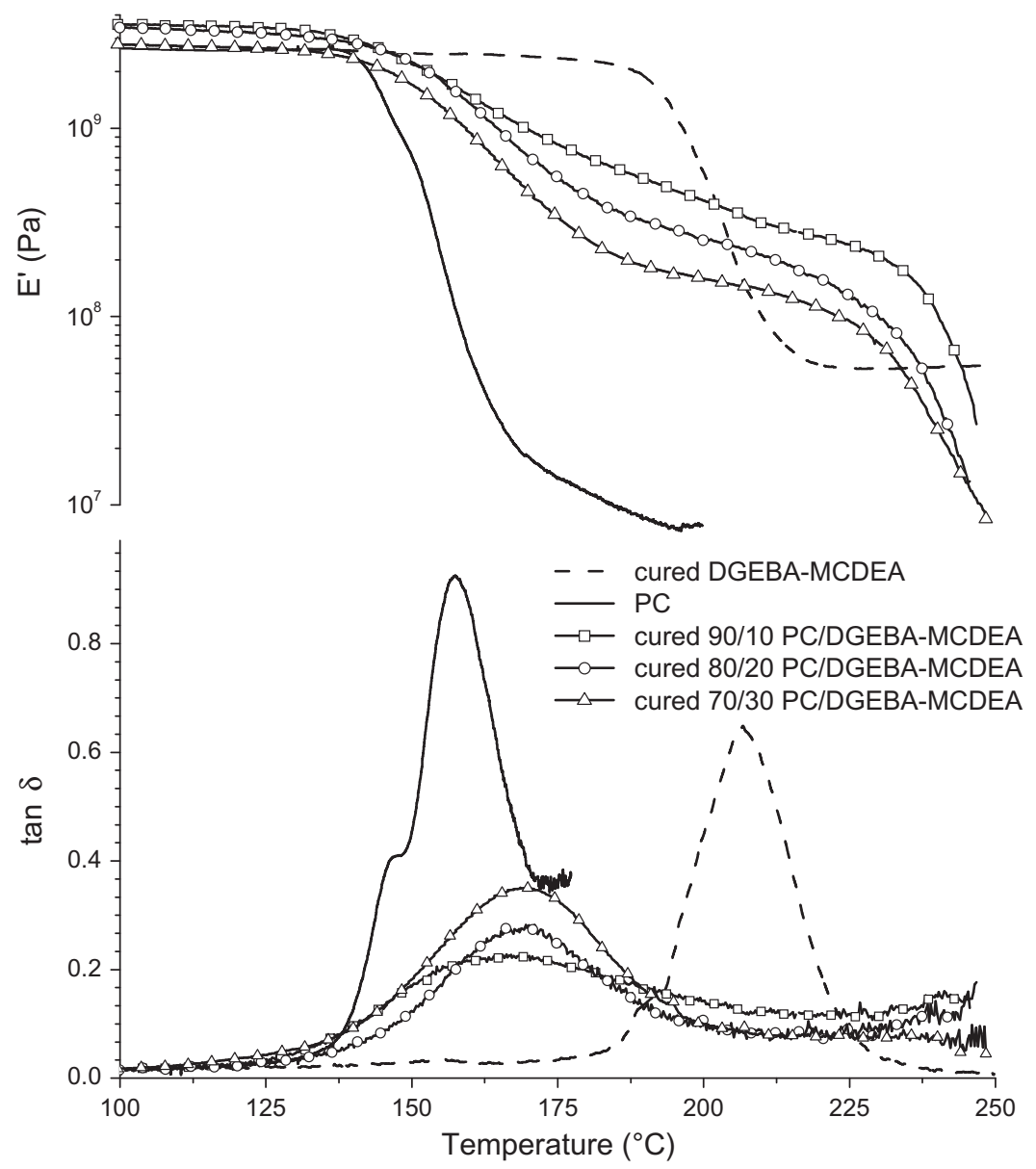

Fig. 10. DMTA of PC, cured $\operatorname{DGEBA}(n=0.04)-\mathrm{MCDEA}$ and various blends of $\mathrm{PC} / \mathrm{DGEBA}(n=0.04)$-MCDEA which had been cured at $150{ }^{\circ} \mathrm{C}$ for $20 \mathrm{~h}$. 
a crystallized PC phase and the cured epoxy network - it is not known how much PC may have resided in this latter phase. As a result of this two phase structure, the blends have unique thermal-mechanical properties as shown in Fig. 10 - the modulus curves of the blends lie in between the PC and cured DGEBA $(n=0.04)-$ MCDEA systems and, even though it drops as the temperature rises above the glass transition of the amorphous phase (which can be generally defined as $\sim 170{ }^{\circ} \mathrm{C}$ from the maximum in $\tan \delta$ ), the modulus does not rapidly decrease until the melting point of the crystalline PC is attained near $240{ }^{\circ} \mathrm{C}$ [19]. This observation is significant in terms of the high temperature applications to which these blends could be put. Takemori [30], has shown that the heat distortion temperature, HDT, (also known as the deflection temperature under load) is approximately located at a modulus of approximately $0.7 \mathrm{GPa}$. On this basis, the HDT of PC is predicted to be $150{ }^{\circ} \mathrm{C}$ while that of the cured blends are greater and range from $164^{\circ}$ and $178^{\circ} \mathrm{C}$. In addition, it is well known [30] that the HDT of the single phase polymers are largely unaffected by the addition of rigid fillers, whereas for two phase polymers (semicrystalline polymers and immiscible polymer blends) fillers can have a large effect. Thus the HDT of PC or of the cured epoxy would not be expected to be greatly affected by addition of fillers, but the HDT of the two phase cured blends would be predicted to be markedly raised by filler addition - for example if the room temperature modulus of these blends was raised by a factor of five - a trivial exercise if fibre or filler reinforcement were used - then cured PC/DGEBA-MCDEA blends with HDTs of $210^{\circ}$ to $240^{\circ} \mathrm{C}$ might be expected. The higher G' of cured PC/DGEBA $(n=0.04)-\mathrm{MCDEA}$ in the rubbery region than that of pure PC suggests the crystallized PC must be much thermal-resistant than the amorphous PC.

Fig. 10 also shows that the $\tan \delta$ curves of the cured $\operatorname{DGEBA}(n=0.04)$-MCDEA blends exhibit broad transitions with maxima between $165^{\circ}$ and $170{ }^{\circ} \mathrm{C}$ which are in between the $T_{g}$ of PC $\left(157^{\circ} \mathrm{C}\right)$ and the cured DGEBA $(n=0.04)-\operatorname{MCDEA}\left(207^{\circ}\right)$ but analysis of this data shows the $T_{g} s$ do not obey the Fox equation [22,23] (Eq. (1)), possibly because the composition of the amorphous polymer in the blend does not have the composition of the overall composition since some of the PC is in the crystalline phase. In addition the amorphous polymer may not be of a single composition but may have a gradient in phase compositions. This latter conclusion is based on the observation that the $\tan \delta$ curves for the blends are low and broad suggesting that the amorphous component is heterogeneous.

At the highest temperatures in Fig. 10, the modulus for each of the cured blends drops dramatically due to melting of the crystalline PC phase but does not appear to approach a rubbery region, despite the fact that the epoxy-amine component is crosslinked. This suggests that, to a large extent, the epoxy-amine phase is not continuous throughout the polymer matrix, but mainly resides in dispersed crosslinked domains, and that the PC is the main continuous phase. After temperature ramping to $250^{\circ} \mathrm{C}$ (above the PC melting temperature) and cooling in the DMTA instrument, the $70 / 30$ specimen of the PC/DGEBA $(n=0.04)$ MCDEA blend was remeasured by DMTA (see Fig. 11). The modulus curve for this re-run specimen does show evi-

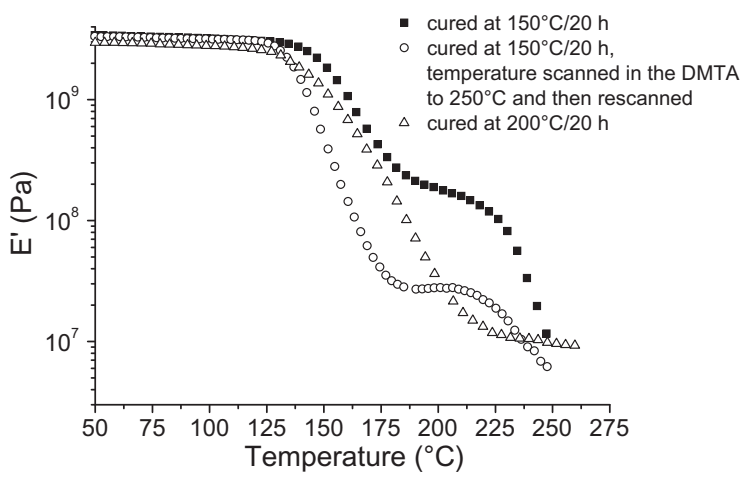

Fig. 11. Storage modulus of the cured $70 / 30 \operatorname{PC} / \operatorname{DGEBA}(n=0.04)-\mathrm{MCDEA}$ blend after cure at $150{ }^{\circ} \mathrm{C} / 20 \mathrm{~h}(\boldsymbol{\square})$ indicating the presence of crystalline PC, and the same specimen measured during a second DMTA experiment $(\bigcirc)$ indicating mainly amorphous material. For comparison, the DMTA behaviour of the same blend but cured at $200^{\circ} \mathrm{C} / 20 \mathrm{~h}(\triangle)$ is also shown and this suggests a completely amorphous polymer.

dence of the melting of PC lamellae by a plateau region near $200{ }^{\circ} \mathrm{C}$ but the modulus in this plateau is about on tenth of that of the original sample, indicating a much lower level of crystallinity. This appears to be due to the difficulty of the PC crystalline lamellae, which were present in the original sample but which were melted during the first DMTA experiment, of being unable to recrystallize upon cooling in the DMTA due to the low PC molecular mobility and the rigidity of the cured epoxy network. These results are not entirely consistent with the hot-stage microscopy data shown in Fig. 8 and the XRD in Fig. 9 which did not indicate any significant crystallinity in the cured 70/30 PC/DGEBA-MCDEA sample after it had been tested in the DMTA. This discrepancy may be caused by the merging of the much smaller crystalline peak with the background in the XRD data and due to the small dimensions of these crystallites which scatter visible radiation less than more perfect crystallites in the hot-stage microscopy study. In order to further verify these explanations, a specimen of the $70 / 30 \mathrm{PC} / \operatorname{DGEBA}(n=0.04)$-MCDEA blend was prepared but instead of curing at $150^{\circ} \mathrm{C}$ where crystallization can occur, this sample was cured at $200^{\circ} \mathrm{C} / 20 \mathrm{~h}$. At $200^{\circ} \mathrm{C}$, the PC in the blend does not crystallize (as shown in Figs. 3 and 8 and reported by Don and Bell [22]) but the epoxy system polymerises quite quickly (see Fig. 1 ) and as a result it is expected that this sample would be amorphous. This is confirmed in Fig. 11 - the specimen cured at $200^{\circ} \mathrm{C}$ has a broad transition region, perhaps caused by the presence of several mixed PC/epoxy network phases, but gives no evidence of crystallinity. Thus it can be concluded that if the epoxy-amine cures slowly at low temperatures, it can act as a solvent which aids crystallization of PC and provides a material with higher thermo-mechanical properties than the amorphous PC and with better stressbearing properties than the cured epoxy between $200^{\circ}$ and $225^{\circ} \mathrm{C}$.

The modulus in the high temperature region for the 70/ $30 \mathrm{PC} / \mathrm{DGEBA}(n=0.04)-\mathrm{MCDEA}$ blend cured at $200^{\circ} \mathrm{C}$ appears to attain a rubbery plateau which is typical of a polymer that has a network structure which is continuous throughout the matrix. In contrast, the behaviour observed 
Table 3

Sol fraction, grafted PC fraction and solvent resistance of PC/DGEBA( $n=0.04)-\mathrm{MCDEA}$ blends cured at $150{ }^{\circ} \mathrm{C} / 20 \mathrm{~h}$.

\begin{tabular}{|c|c|c|c|c|}
\hline $\operatorname{PC} / \mathrm{DGEBA}(n=0.04)-\operatorname{MCDEA}(\mathrm{w} / \mathrm{w})$ & $100 / 0$ & $90 / 10$ & $80 / 20$ & $70 / 30$ \\
\hline Soluble fraction $(\mathrm{w} / \mathrm{w})$ & 1.00 & 0.80 & 0.51 & 0.34 \\
\hline Grafted PC fraction (w/w) & 0 & 0.11 & 0.36 & 0.51 \\
\hline Effect of $\mathrm{CH}_{2} \mathrm{Cl}_{2}$ & Dissolved & $\begin{array}{l}\text { Disintegrated but } \\
\text { did not dissolve }\end{array}$ & $\begin{array}{l}\text { Disintegrated but did } \\
\text { not dissolve }\end{array}$ & $\begin{array}{l}\text { Disintegrated but did } \\
\text { not dissolve }\end{array}$ \\
\hline
\end{tabular}

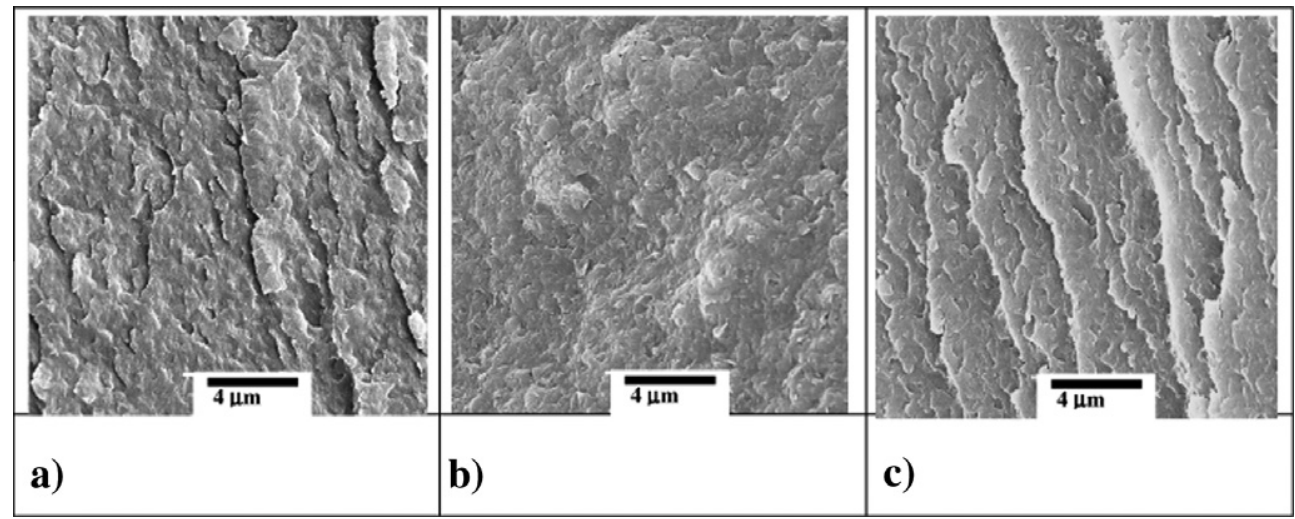

Fig. 12. SEM images of various $\mathrm{PC} / \mathrm{DGEBA}(n=0.04)-\mathrm{MCDEA}$ samples after curing at $150{ }^{\circ} \mathrm{C} / 20 \mathrm{~h}$ and freeze-fracturing: $(\mathrm{a}) 90 / 10 \mathrm{PC} / \mathrm{DGEBA}(n=0.04)-$ MCDEA, (b) 80/20 PC/DGEBA( $n=0.04)-M C D E A$, (c) 70/30 PC/DGEBA( $n=0.04)-\operatorname{MCDEA}$.

when the same blend composition is cured at $150{ }^{\circ} \mathrm{C}$ shows no rubbery plateau, suggesting that the network is not cocontinuous throughout the specimen. Thus it appears that the morphology of the blend can be tuned by selection of the curing temperature because differing morphology and mechanical properties can be obtained depending on the cure temperature.

\subsection{Extraction studies and scanning electron microscopy}

The weight fraction of extractable material from the cured blends is given in Table 3 and this decreases as the fraction of PC in the blend is lowered, as expected. However, the amount of soluble material is lower than the amount of PC in the blend and so some of the PC must be grafted to the epoxy network, presumably as a result of the interchange reaction between the hydroxyl groups in the DGEBA oligomer and the PC carbonate groups as discussed above. Assuming that all of the DGEBA and MCDEA are reacted into the network, the fraction of the PC which is grafted to the network is also tabulated and this is found to increase as the amount of epoxy in the blend is raised, because an increased fraction of the DGEBA oligomer in the blend would be expected to cause greater grafting of the PC to the epoxy network. Although not measured, the trend in this data suggests that for a 60/40 composition the fraction of PC grafted onto the DGEBA oligomer would be more than $50 \%$ and this is considerably higher than the fraction of 0.24 calculated from the molecular weight of the extruded 60/40 PC/DGEBA $(n=0.15)$ blend, and this in turn is higher than that expected if the changes in molecular weight of the $\operatorname{PC} \operatorname{DGEBA}(n=0.04)$ blend had been measured. Presumably this difference exists because the sample studied by GPC had been processed for only $\sim 1 \mathrm{~min}$ at $200{ }^{\circ} \mathrm{C}$, whereas the curing of the 60/40 PC/DGE$\mathrm{BA}(n=0.04)-\mathrm{MCDEA}$ blend was for $20 \mathrm{~h}$ at $150^{\circ} \mathrm{C}$ which could allow more extensive bond interchange reactions via the hydroxyl groups on the $\operatorname{DGEBA}(n=0.04)$ species and with the hydroxyl groups formed during the curing reaction. The studies of Don and Bell [22] on the scission of PC by a similar DGEBA oligomer support this argument.

Table 3 also lists the effect on samples swollen in $\mathrm{CH}_{2} \mathrm{Cl}_{2}$ for three months. None of the PC/DGEBA( $n=0.04)-\mathrm{MCDEA}$ blends dissolved but they did disintegrate into very fine particles with diameters of the order of micrometers as was also found in previous studies of blends of polyvinyl chloride with crosslinkable diallyl phthalate [9]. These particles are probably composed of cured DGEBA $(n=0.04)-$ MCDEA and grafted PC. The fact that the samples disintegrated rather than swelled suggests that either the matrix is uncrosslinked PC or that a only small fraction of the epoxy network is co-continuous with the $\mathrm{PC}$ in the matrix so that, under the solvent-induced stresses, this network breaks into micron-sized particles. These conclusions are consistent with the DMTA data which showed that these blends (cured at $150^{\circ} \mathrm{C}$ ) did not appear to have significant levels of crosslinking throughout the matrix phase.

SEM micrographs of the cured blends (see Fig. 12) indicate a two phase morphology with spherical particles and holes with diameters of the order of $1 \mu \mathrm{m}$ on the fracture surface. This morphology is similar to that observed found in previous studies of blends of polyvinyl chloride with crosslinkable diallyl phthalate [9].

\section{Conclusions}

Blends of PC and a DGEBA oligomer containing hydroxyl groups have been prepared and only a small amount of 
reaction between the DGEBA oligomer's hydroxy group and the PC carbonate group was found by GPC analysis. Use of the epoxy resin as a reactive plasticizer has been shown to greatly reduce viscosity and thus the processing temperature of polycarbonate and $T_{g}$, however it can also greatly accelerated the crystallisation rate of PC, particularly at PC levels of less than $80 \mathrm{wt} . \%$, as shown by rheology, DMTA, XRD, and optical transmission microscopy.

DMTA studies of PC/DGEBA-MCDEA blends cured at intermediate temperatures $\left(150^{\circ} \mathrm{C}\right)$ shows that the original properties of the PC is recovered when the epoxyamine component is polymerised and that materials with heat distortion temperatures greater than that of PC or the epoxy network can be formed and that this behaviour is due to the presence of the crystallized PC phase. However curing the 70/30 PC/DGEBA-MCDEA blend at high temperatures $\left(200^{\circ} \mathrm{C}\right)$ does not allow PC crystallization to occur and so the modulus curve via DMTA shows behaviour intermediate between the PC and DGEBA-MCDEA network.

Extraction studies showed that after curing the PC/DGEBA-MCDEA blends, a significant amount of the PC was grafted to the epoxy network. If this is found to be undesirable it could be reduced by use of pure DGEBA monomer rather than the DGEBA oligomer since much of the grafting reactions probably occurs during the processing stages via the oligomer's hydroxy group. The PC/DGEBA-MCDEA blends disintegrated in solvent but did not dissolve, suggesting that the majority of the crosslinked network is not continuous throughout the matrix. This is confirmed by DMTA studies which show that PC/DGEBA-MCDEA blends cured at $150^{\circ} \mathrm{C}$ do not exhibit a rubbery plateau and SEM micrographs which show a dispersed particulate phase, presumably due to crosslinked epoxy particles and grafted PC.

\section{Acknowledgements}

This project is financially supported by the Australian Research Council with the Grant DP0557737. The authors also would like to thank Ms Laleh Tcharkhtchi and Ms
Arjulizan Rusli for experimental assistance and Mr Victor de Guzman for performing the GPC measurements.

\section{References}

[1] Venderbosch RW, Meijer HEH, Lemstra PJ. Polymer 1995;36: 2903-13.

[2] Venderbosch RW, Meijer HEH, Lemstra PJ. Polymer 1995;36: 1167-78.

[3] Venderbosch RW, Meijer HEH, Lemstra PJ. Polymer 1994;35: 4349-57.

[4] van Pelt WWGJ, Goossens JGP, Meijer HEH, Lemstra PJ. Polymer 2002;43:5699-708.

[5] Jansen BJP, Meijer HEH, Lemstra PJ. Polymer 1999;40:2917-27.

[6] Goosens JGP, Rastogi S, Meijer HEH, Lemstra PJ. Polymer 1998;39: 6577-88.

[7] Liang GG, Cook WD, Sautereau HJ, Tcharkhtchi A. Euro Polym J 2008;44:366-75.

[8] Mounif E, Liang GG, Cook WD, Bellenger V, Tcharkhatchi A. Polym Int 2009;58:954-61.

[9] Liang GG, Cook WD, Sautereau HJ, Tcharkhatchi A. Polymer 2009;50: 2655-63.

[10] Liang GG, Cook WD, Sautereau HJ, Tcharkhatchi A. Polymer 2009;50: 2635-42.

[11] Cook WD, Liang GG, Lu M, Simon GP, Yeo E. Polymer 2007;48: 7291-300.

[12] Venderbosch RW, Meijer HEH, Lemstra PJ. Polymer 1994;35:4349.

[13] Yang Y, Fujiwara H, Chiba T, Inoue T. Polymer 1998;39:2745-50.

[14] Poncet S, Boiteux G, Pascault J-P, Sautereau H, Seytre G, Rogozinski J, et al. Polymer 1999;40:6811.

[15] Venderbosch RW, Peijst T, Meijer HEH, Lemstra PL. Composites A: App Sci Manuf 1996;27A:895-905.

[16] Hernandez M, Sixou B, Duchet J, Sautereau H. Polymer 2007;48: 4075-86.

[17] Don TM, Yeh CH, Bell JP. J Appl Poly Sci 1999;74:2510-21.

[18] Rong MZ, Zheng HM. Polymer 1997;38:269-77.

[19] Brydson JA. Plastics materials. 6th ed. Oxford: Butterworth; 1995.

[20] Lee H, Neville K. Handbook of epoxy resins. New York: McGraw-Hill 1967. pp. 4-29.

[21] Rozenberg BA. Adv Poly Sci 1985;75:113.

[22] Don TM, Bell JP. J Polym Sci A: Polym Chem 1996;34:2103-16.

[23] Fox TG. Bull Am Phys Soc 1956;1:123-5.

[24] Fox TG, Flory PJ. J Appl Phys 1950;21:581.

[25] Fox TG, Flory PJ. J Polym Sci 1954;14:315.

[26] Cheah K, Cook WD. Polym Eng Sci 2003;43:1727-39.

[27] Sohn S, Alizadeh A, Marand H. Polymer 2000;41:8879-86.

[28] Mercier JP, Legras R. J Polym Sci Part B: Polym Lett 1970;8:645-50.

[29] Liao X, Wang J, Li G, He J. J Polym Sci Part B: Polym Phys 2004:42:280-5

[30] Takemori T. Polym Eng Sci 1979;19:1104-9. 